\title{
Possible Phason-Polaron Effect on Purely One-Dimensional Charge Order of $\mathrm{Mo}_{6} \mathrm{Se}_{6}$ Nanowires
}

\author{
Xing Yang $\odot,{ }^{1, *}$ Jing-Jing Xian,,${ }^{1, *}$ Gang Li, ${ }^{2}$ Naoto Nagaosa, ${ }^{3,4}$ Wen-Hao Zhang, ${ }^{1}$ Le Qin, ${ }^{1}$ \\ Zhi-Mo Zhang, ${ }^{1}$ Jing-Tao Lü®${ }^{1, \dagger}$ and Ying-Shuang Fu $\odot^{1, \$}$ \\ ${ }^{1}$ School of Physics and Wuhan National High Magnetic Field Center, \\ Huazhong University of Science and Technology, Wuhan 430074, China \\ ${ }^{2}$ School of Physical Science and Technology, ShanghaiTech University, Shanghai 200031, China \\ ${ }^{3}$ RIKEN Center for Emergent Matter Science (CEMS), Wako, Saitama 351-0198, Japan \\ ${ }^{4}$ Department of Applied Physics, The University of Tokyo, Tokyo 113-8656, Japan
}

(Received 14 February 2020; revised 12 June 2020; accepted 28 July 2020; published 17 September 2020)

\begin{abstract}
In one-dimensional (1D) metallic systems, the diverging electron susceptibility and electron-phonon coupling collaboratively drive the electrons into a charge density wave (CDW) state. However, a strictly 1D system is unstable against perturbations, whose effect on CDW order requires clarification ideally with altered coupling to surroundings. Here, we fabricate such a system with nanowires of $\mathrm{Mo}_{6} \mathrm{Se}_{6}$ bundles, which are either attached to edges of monolayer $\mathrm{MoSe}_{2}$ or isolated freely, by postannealing the preformed $\mathrm{MoSe}_{2}$. Using scanning tunneling microscopy, we visualize charge modulations and CDW gaps with prominent coherent peaks in the edge-attached nanowires. Astonishingly, the CDW order becomes suppressed in the isolated nanowires, showing CDW correlation gaps without coherent peaks. The contrasting behavior, as revealed with theoretical modeling, is interpreted as the effect of phason polarons on the 1D CDW state. Our work elucidates a possibly unprecedented many-body effect that may be generic to strictly 1D system but undermined in a quasi-1D system.
\end{abstract}

DOI: 10.1103/PhysRevX.10.031061

\section{INTRODUCTION}

When electrons are confined in an atomic chain, correlations among them get enhanced, causing instability of the Fermi surface due to its perfect nesting and reduced screening [1]. This instability makes the systems susceptible to electron-electron and electron-phonon interactions, driving them into a rich variety of exotic correlated quantum states that are distinguished from $3 \mathrm{D}$, such as the Tomonaga-Luttinger liquid, spin density waves, and charge density waves (CDWs). In the CDW state, lattice symmetry is spontaneously broken via a Peierls distortion mechanism dictated by the electron-phonon coupling [2]. The electron density acquires a spatial modulation corresponding to twice the Fermi wave vector $\left(k_{F}\right)$ and concomitantly opens a gap around the Fermi energy $\left(\varepsilon_{F}\right)$. However, quantum and thermal fluctuations in a strictly 1D

\footnotetext{
*These authors contributed equally to this work. jtlu@hust.edu.cn

¥yfu@hust.edu.cn
}

Published by the American Physical Society under the terms of the Creative Commons Attribution 4.0 International license. Further distribution of this work must maintain attribution to the author(s) and the published article's title, journal citation, and DOI.
Subject Areas: Condensed Matter Physics

Materials Science, Nanophysics system induce uncertainty in nuclear positions of the same order as that of the Peierls distortion [3], which tend to destroy the CDW coherence. Indeed, most experimental CDW orders are observed in quasi-1D systems [4-7] that are ensembles of chains contained in either a bulk $3 \mathrm{D}$ or a surface 2D form. Their interchain interaction is argued to stabilize the CDW state. While intriguing properties of 1D CDWs such as solitons [8-11] are revealed, the interchain interaction inevitably causes complications. It is desirable to experimentally examine its existence in a truly 1D system. Although recent experiments report CDW orders in single silicide nanowires [12] and a mirror twin boundary of monolayer $\mathrm{MoSe}_{2}$ [13] that are indeed electronically isolated 1D systems, they are still coupled to the lattice of supporting surroundings, whose phonons are essentially 2D. Thus, a 1D system with altered coupling to its surroundings is essential to uncover the embedded properties in inherent CDW order.

Creating such a system is challenging, because it should have 1D electronic character with reasonable regularity, defect-free from disorder, and retain its 1D electronic property against altered coupling to the surroundings. Our strategy is to use transition metal monochalcogenide (TMM) nanowires, which have monolayer thickness and nanometer width and show metallic conductivity [14-17]. TMM is a polymorph of transition metal dichalcogenides 
(TMDs), which are widely studied due to their direct-band gaps with spin and valley polarization [18] at the single-layer limit. Because of the polymorph, TMM nanowires can be obtained by judiciously tuning the stoichiometry and even achieving contacts to TMDs. However, TMM nanowires synthesized with previous methods suffer from either shape irregularities or fixed coupling to surroundings [19-25].

Here, we present an approach for fabricating TMM $\mathrm{Mo}_{6} \mathrm{Se}_{6}$ nanowires by postannealing the preformed TMD $\mathrm{MoSe}_{2}$ monolayers prepared with molecular beam epitaxy [26] on a graphene-covered $\mathrm{SiC}(0001)$ substrate [27], producing straight nanowires with monolayer height and well-defined width. The nanowires are either attached to the $\mathrm{MoSe}_{2}$ edges or isolated freely, allowing altered coupling to the environment. Equally importantly, the graphene substrate has a negligible interaction with the supported nanowires. Both conditions promise a system ideal for the study of purely $1 \mathrm{D}$ CDW order.

\section{RESULTS}

\section{A. Morphology and electronic structure of $\mathrm{Mo}_{6} \mathrm{Se}_{6}$ nanowires}

Figure 1(a) shows the morphology of $\mathrm{MoSe}_{2}$ monolayer islands and a small fraction of bilayer islands all decorated with $\mathrm{Mo}_{6} \mathrm{Se}_{6}$ nanowires at the edges. Isolated nanowires bridging $\mathrm{MoSe}_{2}$ monolayer islands are also seen. Statistics over 30 nanowires indicates that their width is
$3.35 \pm 0.26 \mathrm{~nm}$ and uniform throughout individual ones. This result reflects the nanowires are $\mathrm{Mo}_{6} \mathrm{Se}_{6}$ bundles containing mostly three wires or four wires in parallel. An enlarged image of the bright edge displays the atomic resolution of the nanowire [Fig. 1(b)], showing a lattice constant of $0.44 \mathrm{~nm}$ along the nanowire. Its atomic resolution image is reproduced by our simulated STM image with density functional theory (DFT) based on the crystal structure of a four-wire $\mathrm{Mo}_{6} \mathrm{Se}_{6}$ bundle [Fig. 1(b) and Supplemental Note 1 [28]]. The atomic structure of such TMM wires prepared with a similar method is also bserved with a transmission electron microscope [24]. There is a moire period approximately $1.3 \mathrm{~nm}$ along the nanowires, implying their interface with $\mathrm{MoSe}_{2}$ is atomically smooth, and a larger one at approximately $5 \mathrm{~nm}$ that is likely from imperfect moiré overlapping [Figs. S4(a) and S5 [28] ].

Next, we show their tunneling spectra. While $\mathrm{MoSe}_{2}$ has

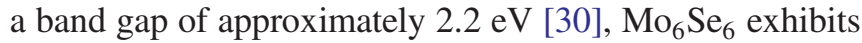
increased conductance with multiple peaks below $\varepsilon_{F}$, a relatively flat but finite conductance above $\varepsilon_{F}$, and an enhanced peak at approximately $1.6 \mathrm{eV}$ with a splitting of approximately $0.2 \mathrm{eV}$ [Figs. 1(c) and S6 [28] ]. The edgeattached and isolated nanowires exhibit similar spectra, indicating their electronic structure of 1D nature is well conserved against the edge attachment to the $\mathrm{MoSe}_{2}$ layer. Those spectroscopic features are captured by our DFT calculations of both a single $\mathrm{Mo}_{6} \mathrm{Se}_{6}$ wire [Fig. 1(d)] and a

\author{
(a)
}

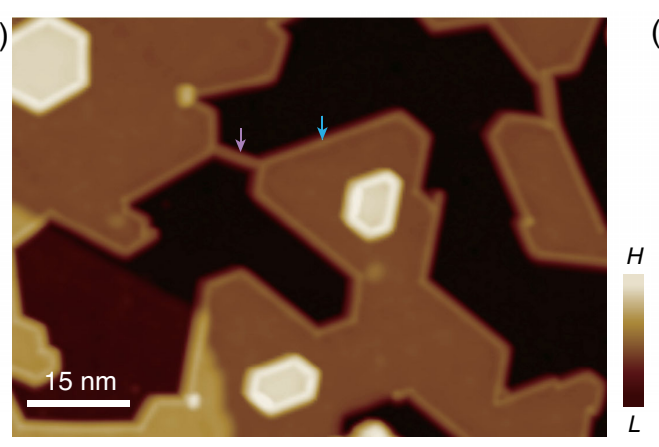

(b)

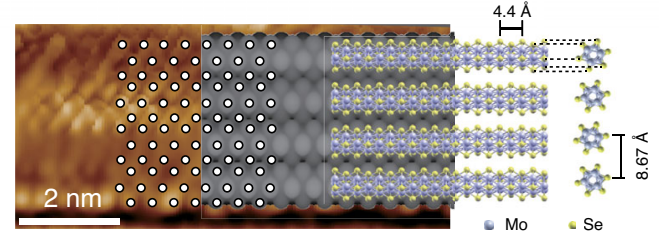

(c)
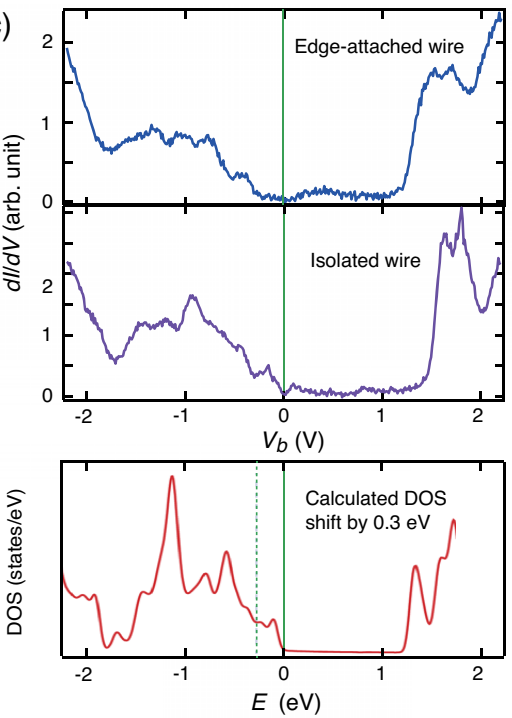

(d)

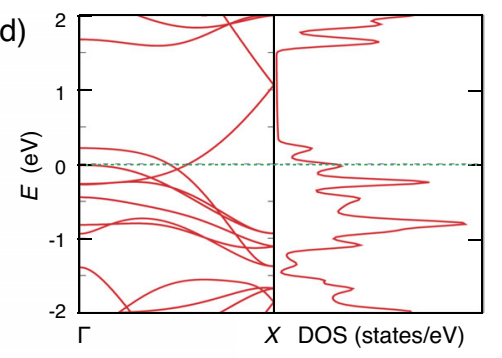

(e)

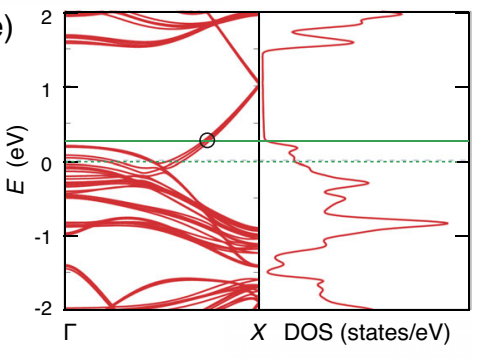

FIG. 1. Morphology and electronic structure of $\mathrm{Mo}_{6} \mathrm{Se}_{6}$ nanowires. (a) STM topography $\left(V_{s}=2.2 \mathrm{~V}, I_{t}=10 \mathrm{pA}\right)$ of Mo $\mathrm{Se}_{6}$ nanowires. An exemplified isolated (edge attached to $\mathrm{MoSe}_{2}$ monolayer) nanowire is indicated with a purple (cyan) arrow. (b) Atomic resolution image $\left(V_{s}=150 \mathrm{mV}, I_{t}=100 \mathrm{pA}\right)$ of an edge-attached nanowire, which is superimposed with the crystal structure of a four-wire bundle [top view (left) and side view (right)] and its DFT-simulated STM image. The white dots mark selected positions of the imaged atoms. (c) Typical tunneling spectra of an edge-attached (blue curve) and an isolated (purple curve) nanowire of four-wire bundle $\left(V_{s}=2.2 \mathrm{~V}, I_{t}=100 \mathrm{pA}, V_{\text {mod }}=14.14 \mathrm{mV}_{\mathrm{rms}}, T=77 \mathrm{~K}\right)$. (d),(e) DFT-calculated band structure and density of states (DOS) of a single $\mathrm{Mo}_{6} \mathrm{Se}_{6}$ wire (d) and a three-wire bundle (e), whose crystal structure is the same as that in (b). The solid and dashed green lines in (c)-(e) mark $\varepsilon_{F}$ of the measured and calculated nanowires, respectively. The black circle marks $k_{F}$. The DOS of (e) is shown in (c) for comparison. 
bundle containing $2-5$ wires [three-wire bundle exemplified in Fig. 1(e)], demonstrating their bands are not substantially modified by the bundled structure. Comparison with the experiment reveals the nanowires are electron-doped approximately $0.3 \mathrm{eV}$ by the substrate [Fig. 1(c)] resulting in a $k_{F} \sim 5.35 \mathrm{~nm}^{-1}$ [Fig. 1(e)].

\section{B. CDW of $\mathrm{Mo}_{6} \mathrm{Se}_{6}$ nanowires}

Intriguingly, spectra of a typical edge-attached nanowire [Fig. 2(a)] at $4.4 \mathrm{~K}$ reveal a salient gap spreading the entire nanowire (Figs. 2(b) and S4(b) [28] ]. The gaps exhibit sharp coherence peaks with a gap size $(2 \Delta)$ of approximately $92 \mathrm{meV}$ [Fig. 2(c)]. Statistics over 17 nanowires indicate an average $2 \Delta$ of $72.5 \mathrm{meV}$ with a standard deviation of $29.3 \mathrm{meV}$, which is on a similar scale as that reported in the $\mathrm{MoSe}_{2}$ mirror twin boundary [13]. The $2 \Delta$ variation implies their different couplings to the $\mathrm{MoSe}_{2}$ layers coming from the edge-attachment configurations. The observed gap cannot stem from disorder-induced electron localization [31,32], because it has a $U$ shape with coherence peaks and a spatially identical size throughout each nanowire. To explore its CDW origin, we examine the real-space conductance of the nanowire around energies of the coherence peaks. There exhibits periodic modulations of $0.64 \pm 0.04 \mathrm{~nm}$, which coincide with the calculated CDW period of $\pi / k_{F}$ [Fig. 1(e)] and are in antiphase for the filled and empty states, respectively [Fig. 2(d) and another dataset in Figs. S4(b)-S4(d)]. These features are consistent with the convention of CDW order. This ascription is augmented by elevating the temperature, where the CDW gap becomes intrinsically suppressed [Figs. 2(e) and S7 and Supplemental Note 2 [28] ].

The CDW order in edge-attached nanowires has several properties. First, the CDW modulation, whose measured period $(0.64 \pm 0.04 \mathrm{~nm})$ is 1.44 times the lattice constant of $\mathrm{Mo}_{6} \mathrm{Se}_{6}(0.444 \pm 0.013 \mathrm{~nm})$, is incommensurate with the lattice and also found barely influenced by the strainreleasing defects [Fig. S4(e) [28] ]. The incommensurate band filling is from the electron charge transfer from the substrate to the nanowires. Second, there exists conductance inhomogeneity along the nanowire that correlates with the imperfect moiré overlapping. The spatial scale is consistent with the CDW correlation length of $\hbar v_{F} / 2 \Delta \sim$ $2.5 \mathrm{~nm}$ [33] [Figs. 2(b) and S4(b)]. Third, a series of satellite peaks of approximately $15 \pm 2 \mathrm{meV}$ spacing appear next to the coherence peaks [Figs. 2(c) and 2(d)], which are ascribed to a phonon mode [13]. Fourth, the supporting graphene has negligible influence on the CDW order (Supplemental Note 3 [28]).

Interestingly, the isolated nanowire also exhibits a spectroscopic gap around $\varepsilon_{F}$ at $4.4 \mathrm{~K}$ [Figs. 3(a) and 3(b)], which features enhanced conductance, related to an adjacent electronic state, below the lower gap edge, and powerlaw-shaped onset of the conductance at the upper gap edge. While the gap shape shows some difference at each spectrum along the nanowire, they all have similar gap size (approximately $130 \mathrm{meV}$ ) except at both end contacts and surprisingly no coherence peaks [Fig. 3(c)]. There is no charge modulation along the nanowire, either [Fig. 3(b)]. A similar spectroscopic gap is observed in ten isolated nanowires of different lengths. The gap is off-centered from $\varepsilon_{F}$ that may (a)

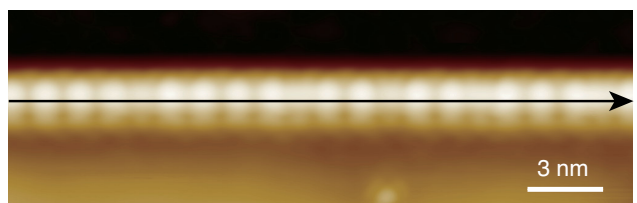

(b)

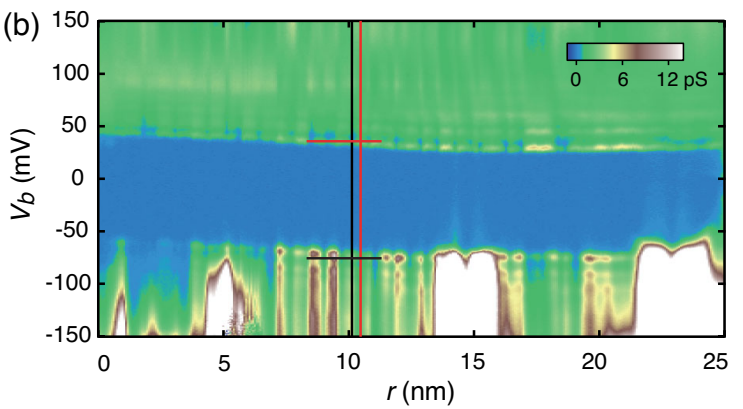

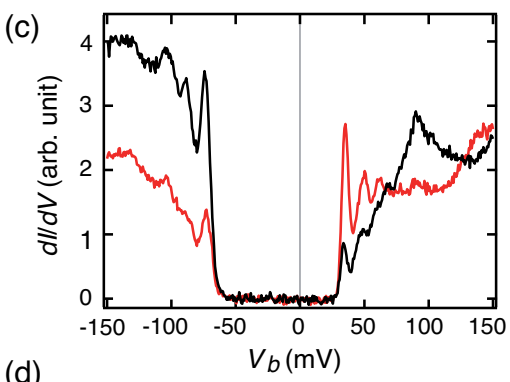

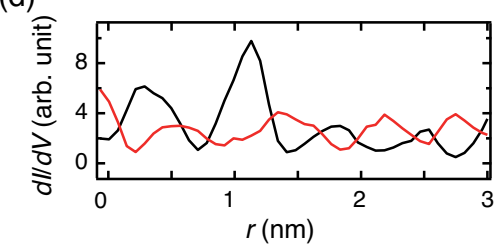

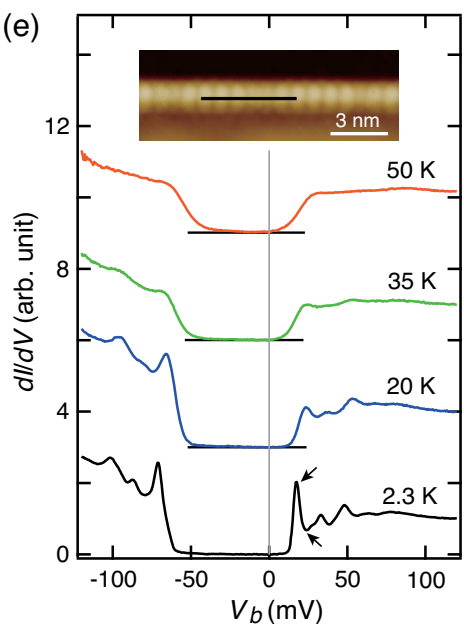

FIG. 2. CDW state of edge-attached $\mathrm{Mo}_{6} \mathrm{Se}_{6}$ nanowire. (a) STM image $\left(V_{s}=600 \mathrm{mV}, I_{t}=10 \mathrm{pA}\right)$ of an edge-attached $\mathrm{Mo}_{6} \mathrm{Se}_{6}$ nanowire (four-wire bundle). (b) $2 \mathrm{D}$ conductance plot taken along the black line in (a) $\left(V_{s}=150 \mathrm{mV}, I_{t}=100 \mathrm{pA}\right.$, $V_{\text {mod }}=2.12 \mathrm{mV}_{\text {rms }}, T=4.4 \mathrm{~K}$ ). (c) Two typical spectra showing a CDW gap. The black (red) curve is extracted from (b) along the vertical black (red) line, corresponding to two adjacent conductance extrema. (d) Line profiles of the conductance plot in (b) along the horizontal red and black line, respectively. (e) Tunneling spectra of an edge-attached nanowire (three-wire bundle) at different temperatures $\left(V_{s}=150 \mathrm{mV}, I_{t}=100 \mathrm{pA}, V_{\bmod }=1.41 \mathrm{mV}_{\mathrm{rms}}\right)$, whose image $\left(V_{s}=500 \mathrm{mV}, I_{t}=10 \mathrm{pA}\right)$ is shown in the inset. Each spectrum is an average of the spectra along the black line $(5 \mathrm{~nm}$ long) of the nanowire. The spectra are offset vertically for clarity. Zero conductance for each spectrum is marked with a black line. 
(a)
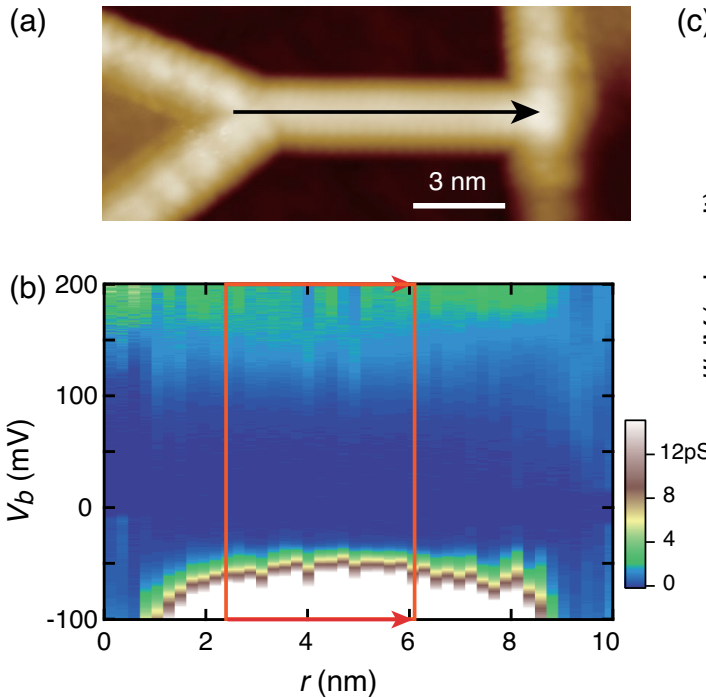

(c)

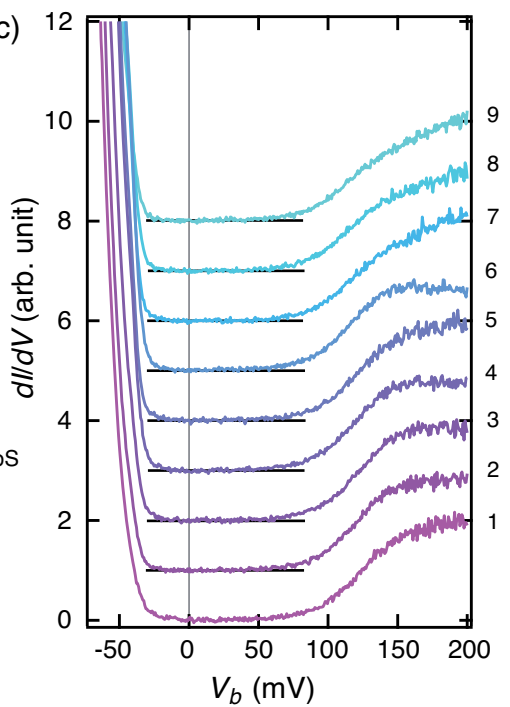

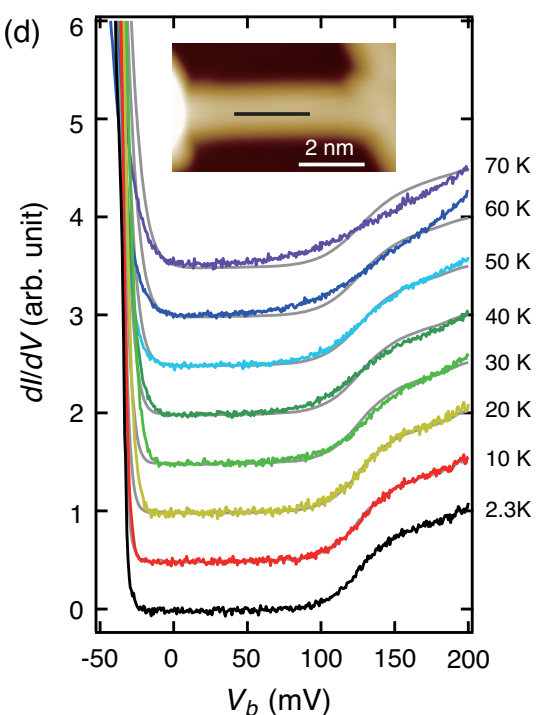

FIG. 3. CDW correlation in isolated $\mathrm{Mo}_{6} \mathrm{Se}_{6}$ nanowire. (a) STM topography $\left(V_{s}=0.5 \mathrm{~V}, I_{t}=20 \mathrm{pA}\right)$ of an isolated $\mathrm{Mo}_{6} \mathrm{Se}_{6}$ nanowire (three-wire bundle). (b) $2 \mathrm{D}$ conductance plot taken along the black line in (a) $\left(V_{s}=200 \mathrm{mV}, I_{t}=100 \mathrm{pA}\right.$, $V_{\text {mod }}=1.41 \mathrm{mV}_{\mathrm{rms}}, T=4.4 \mathrm{~K}$ ). (c) Tunneling spectra extracted from the red rectangle of (b) in an equally spaced manner. The black lines mark the zero conductance for each spectrum. (d) Tunneling spectra of an isolated $\mathrm{Mo}_{6} \mathrm{Se}_{6}$ nanowire (three-wire bundle), whose image $\left(V_{s}=2.2 \mathrm{~V}, I_{t}=10 \mathrm{pA}\right)$ is shown in the inset, at different temperatures $\left(V_{s}=200 \mathrm{mV}, I_{t}=50 \mathrm{pA}\right.$, $\left.V_{\text {mod }}=1.41 \mathrm{mV}_{\text {rms }}\right)$. Each spectrum is an average of the spectra taken along the black line (2.3 nm long) of the inset. For comparison, the spectra are superimposed with the simulated spectra (gray curves) of $2.3 \mathrm{~K}$ with a thermal broadening effect at each temperature. The spectra in (c) and (d) are offset vertically for clarity.

appear at either side (Fig. S10 [28]) and gets smeared intrinsically with increasing temperature [Fig. 3(d)]. Since those characters are shared by the CDW gap of edge-attached nanowires, we are led to relate the spectroscopic gap of the isolated nanowire to CDW correlation. The vanishing oscillatory electron density with the position itself in the presence of the well-defined gap strongly suggests the quantum disorder state due to the presence of phason. Therefore, it is reasonable to assume the incommensurate phason. The CDW correlated state without long-range coherent order $[34,35]$ is analogous to the pseudogap in high-temperature superconductors [36] and disordered superconductors [37], where the Cooper pairs are localized without forming longrange coherence.

\section{DISCUSSION}

\section{A. Possible reasons for impacting the CDW coherence}

Several factors can impact the coherence of CDW order. Atomic disorder can destroy the phase coherence [38]. This factor, however, can be disregarded, as the isolated nanowire has no defect-associated disorder. Finite length of the nanowires could affect the CDW coherence. This possibility is ruled out, because the isolated nanowires are connected to the edge-attached ones, which are long along the $\mathrm{MoSe}_{2}$ edges. Quantum and thermal fluctuations of acoustic phonons and phason of the CDW, which correspond to atomic motions near the zero momentum and $2 k_{F}$, respectively, act as a random potential to the CDW order and can be treated as an effective lattice disorder, destroying the CDW phase coherence [39,40]. This scenario predicts the finite density of states within the gap together with the broadened peak at the edge of the gap, and eventually the gap features vanish in the strong fluctuation limit. This possibility is distinct from the observation in the present study, where the gap remains clearly defined, and only the singularity at the edge is reduced for the case of isolated wire.

\section{B. Phason-polaron model on CDWs of different dimensionality}

When an electron (or a hole) from the STM tip is injected into the nanowire, it may preferentially shake up gapless phonons or phasons, resulting in a polaronic coupling between the injected electron and the phonon [41] or phason. Such a polaronic effect drastically perturbs the otherwise long-rang ordered CDW state [35]. As is formulated below, the polaronic coupling strength to an electron phason is an order of magnitude larger than that of the electron-acoustic-phonon coupling, changing the singularity of the CDW gap spectrum. Moreover, the CDW spectrum exhibits intriguing dependence on the dimensionality of the phason, due to its different density of states between $1 \mathrm{D}$ and 2D.

We first focus on the polaronic effect of electron-phason coupling on the CDW order and start from the electron 
mean field Hamiltonian for the CDW state (Supplemental Note $4.1[28])$

$$
H=\sum_{i=1}^{2 N} \sum_{k} E_{k}\left(a_{k i}^{\dagger} a_{k i}-b_{k i}^{\dagger} b_{k i}\right)
$$

with $E_{k}=\sqrt{\left(v_{F} k\right)^{2}+\Delta^{2}}$. The CDW gap $\Delta$ is given by $\Delta=g u_{0} / \sqrt{L}$ with $g$ the electron-phonon coupling parameter, $u_{0}$ the condensed part of the phonon, and $L$ the length of the chain. The wave vector $k$ is measured with respect to the Fermi level, $a_{k i}$ and $b_{k i}$ are given by the proper linear combinations of the electron destruction operator $c_{i, k+k_{F}}$ and $c_{i, k-k_{F}}, v_{F}$ and $k_{F}$ are the Fermi velocity and wave vector, respectively, and the index $i$ includes both the spin and the chain indices, with the number of chains in the bundle $N$. Next, we write the order parameter $\Delta=|\Delta| e^{i \phi}$ with amplitude $|\Delta|$ and phase $\phi$. For incommensurate CDW as in our case, the low-energy excitation is the gapless phason [Fig. 4(a)]. By introducing fluctuations of $\phi$, we obtain the phason Lagrangian given by

$$
L_{\text {phason }}=N \int d x\left(\left[\frac{\hbar}{4 \pi v_{F}}+\rho u_{0}^{2}\right]\left(\partial_{\tau} \phi\right)^{2}+\frac{\hbar v_{F}}{4 \pi}\left(\partial_{x} \phi\right)^{2}\right) .
$$

This form is identical to that of the acoustic phonon with a linear energy dispersion. The renormalized phason mass density is $\rho_{P}=2 N\left(\rho+4 \pi v_{F} u_{0}^{2} / \hbar\right)$ with $\rho$ the atomic mass density of one chain, and the phason velocity is $c \simeq \sqrt{N \hbar v_{F} /\left(2 \pi \rho_{P} u_{0}^{2}\right)}$. The coupling of the phason to the electron can be written as

$$
H_{\mathrm{int}}=\frac{1}{\sqrt{L}} \sum_{i=1}^{2 N} \sum_{k, q} M_{q}\left(b_{q}+b_{-q}^{\dagger}\right) a_{k+q i}^{\dagger} a_{k i},
$$

(a)

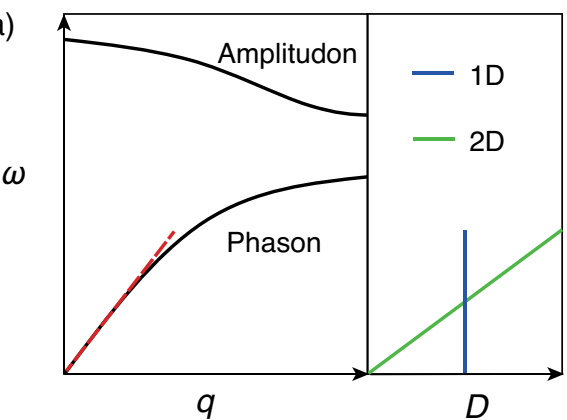

(b)

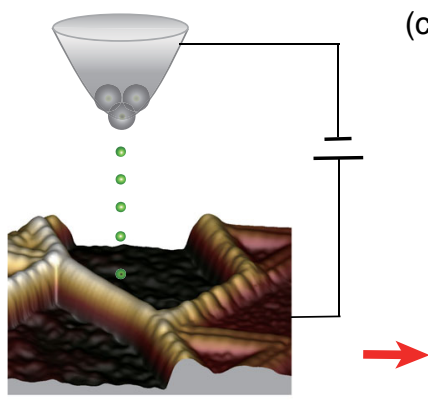

(c)

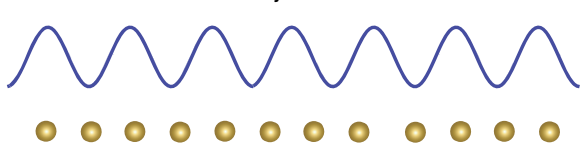

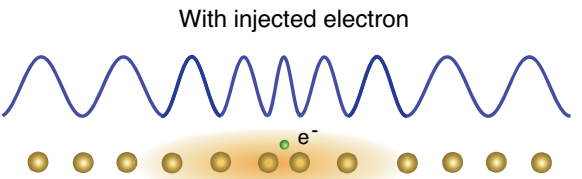

(d)

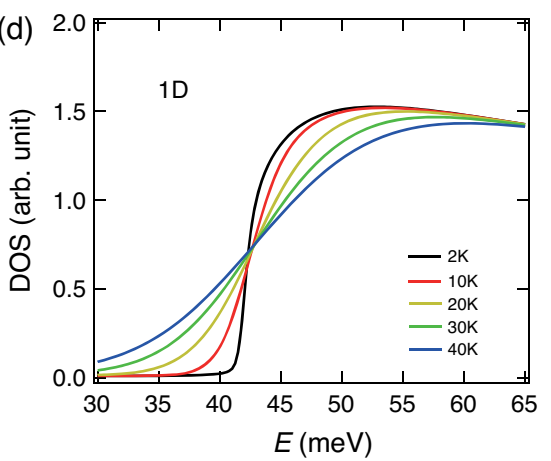

(e)

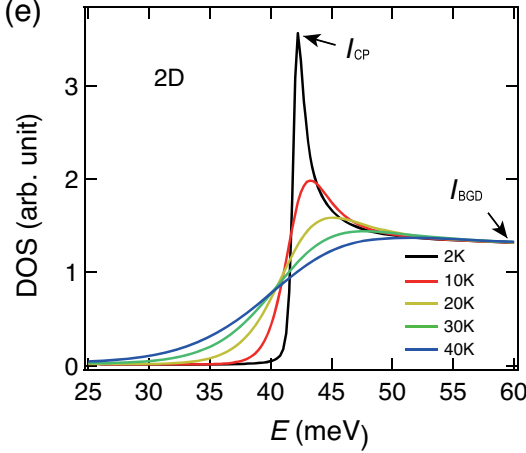

(f)

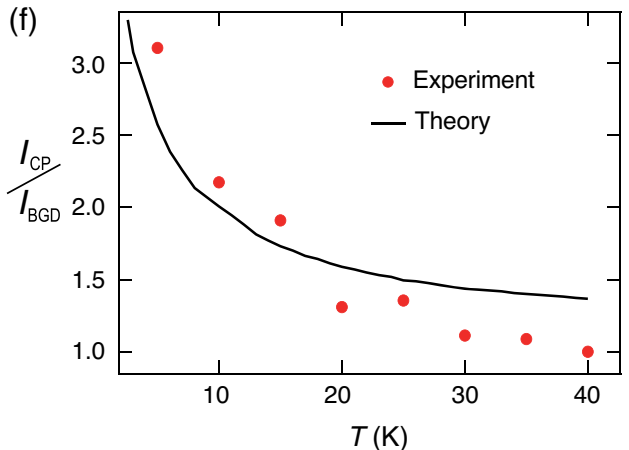

FIG. 4. Phason-polaron effect on a 1D CDW state. (a) Dispersion relation $\omega(q)$ of phason and amplitudon in an incommensurate CDW system (left). The red line is a linear fit of the low-energy phason dispersion, whose density of states $D(\omega)$ for 1D and 2D phasons are a constant and linear with energy, respectively (right). (b) Schematic of the STM experimental configuration. The green balls represent tunneling electrons between the tip and the nanowire. (c) Schematics showing the formation of a phason polaron. Without additional electrons injected into an atomic chain (orange balls), its incommensurate CDW wave (blue curve with regular periodicity) is an almost long-range-ordered state with a power-law correlation function (upper). When a tunneling electron or hole (green ball) injects into or out of the CDW from the STM tip (lower), the atoms in the chain adjust their local positions temporally in response to the additional electron (orange shaded area around the displaced orange balls). Concomitantly, this adjustment shakes up low-energy phasons (blue curve with locally squeezed periodicity). The electron-phason complex forms a phason polaron. (d),(e) Simulated DOS showing spectra of 1D (d) and $2 \mathrm{D}$ phason polarons (e) after considering thermal broadening, corresponding to the cases of isolated and edge-attached nanowires, respectively. For calculating the phason-polaron CDW spectra, we choose $\gamma=0.8, \hbar \omega_{c}=10 \mathrm{meV}, \Delta=42 \mathrm{meV}$, and damping energy $=0.1 \mathrm{meV}$. Only empty states of the spectra are shown for clarity. (f) Comparison between the experimental and theoretical ratios of intensity between the coherence peak $\left(I_{\mathrm{CP}}\right)$ and the background $\left(I_{\mathrm{BGD}}\right)$ [black arrows in (e) and Fig. 2(e)] in the 2D case. 
where $M_{q}=g\left(i \hbar v_{F} q / 2 \Delta\right) \sqrt{\hbar / 2 \rho_{P} \omega_{q}}$. This coupling Hamiltonian shares a similar form as the standard electronacoustic-phonon interaction with an important extra factor $\hbar v_{F} q / 2 \Delta$, because the CDW introduces a rapid change of the electronic states within the narrow range $\xi^{-1} \sim$ $\left(\Delta / \varepsilon_{F}\right) a^{-1}$ in the wave number space with $a$ being the lattice constant and $\xi$ being the coherence length of the CDW. Hence, the coupling to a phason with wave number $q$ is proportional to $\xi q$, while that of the acoustic phonon to $a q$. This proportionality results in a much stronger electronphason coupling than the electron-acoustic-phonon coupling. Therefore, we consider only the electron-phason coupling effect on the spectrum of the CDW gap. Upon injection of a single electron or hole to the CDW state from a STM tip [Fig. 4(b)], the strong electron-phason coupling effect formulated in Eqs. (1)-(3) results in the formation a new quasiparticle dubbed a phason polaron [Fig. 4(c)], where the electrons are dressed by phasons, in analogy with a standard polaron, where electrons are dressed by phonons. Actually, this enhancement of phason coupling is recognized in a previous theoretical study [35]. Our work provides a clear observation of its dependence on the phason dimensionality.

The phason-polaron spectrum can be obtained following the standard procedure as the polaronic coupling to an acoustic phonon (Supplemental Note 4.2 [28]). This procedure gives the spectral function of the 1D phason polaron as $A(\omega) \propto(\omega-\Delta)^{\gamma-1 / 2}$, with $\gamma$ the dimensionless coupling constant. Our estimation of $\gamma \sim 3$ based on parameters from DFT calculations (Supplemental Note 4.3 [28]) gives its upper bound and is consistent with the suppression of the diverging behavior of $A(\omega)$ at $\omega \sim \Delta$. This result is in sharp contrast to the case of a $2 \mathrm{D}$ phason polaron, which gives $A(\omega) \propto e^{-\gamma}(\omega-\Delta)^{-1 / 2}$. Namely, the singularity remains with reduced magnitude. This difference originates from the different dimensionality of the phason density of states, which are constant and linear with energy in 1D and 2D cases, respectively [Fig. 4(a)]. Therefore, the phason density of states of 1D is much larger than that of 2D at low energy, resulting in an enhanced phason-polaron effect in 1D.

With a cutoff phason energy $\hbar \omega_{c}=10 \mathrm{meV}$, which is set by the temperature range of the experiment, and a fitting parameter $\gamma=0.8$, the numerical CDW gaps in the presence of a $1 \mathrm{D}$ and $2 \mathrm{D}$ phason polaron including the thermal broadening effect [Figs. 4(d) and 4(e)] nicely reproduce the experimental features considering the model's simplicity. This phason-polaron scenario can also predict the finitetemperature effect, which in the $2 \mathrm{D}$ case gives $A(\omega) \propto$ $(\omega-\Delta)^{2 \gamma\left(k_{B} T / \hbar \omega_{c}\right)-1 / 2}$ with $k_{B}$ being the Boltzmann constant (Supplemental Note 4.2 [28]). Therefore, it is expected that the singularity of $A(\omega)$ disappears when $k_{B} T>k_{B} T_{c}=\hbar \omega_{c} /(4 \gamma)$, giving $T_{c} \sim 36 \mathrm{~K}$. The temperature dependence of $A(\omega)$, which is quantified by the ratio of intensity between the coherence peak and the background, seems consistent with this expectation [Fig. 4(f) [28] ], giving another support for our picture. Note that our model is based on gapless phasons and assumes that the wires are infinitely long, which are not ideal in experiment. CDW pinning by disorder or commensurate lattice can introduce a phason gap [2]. Moreover, the finite length of the isolated wire introduces a crossover energy scale between the 1D and 2D phason. We evaluate that their influence on phasons is negligible (Supplemental Note 5 [28]).

\section{Outlook}

Our study envisions several future studies. The phasonpolaron effect suggested here should be generic to strictly 1D CDW states, which can be examined in more experimental systems. $\mathrm{Mo}_{6} \mathrm{Se}_{6}$ bundles with increased width, on the other hand, are expected to induce a dimensional crossover from strictly $1 \mathrm{D}$ to quasi-1D, whose influence on the CDW states can be studied systematically. Moreover, the polaronic effect with different dimensionality may also interplay with other correlated states in 1D system, such as spin density waves, Tomonaga-Luttinger liquid [42], etc., which opens up new directions for in-depth investigations.

\section{MATERIALS AND METHODS}

\section{A. Sample preparation \\ 1. $\mathrm{SiC}(0001)$ substrate}

The SiC substrate (MTI corporation) is first degassed at $900 \mathrm{~K}$ for at least $3 \mathrm{~h}$ to remove organic adsorbates. Then, it is flashed to $1220 \mathrm{~K}$ for $2 \mathrm{~min}$ while facing a $\mathrm{Si}$ source heated at $1470 \mathrm{~K}$. Five cycles of similar flashing procedures are performed. Subsequently, the Si source is turned off. And the $\mathrm{SiC}$ is flashed to a higher temperature of $1670 \mathrm{~K}$ for $5 \mathrm{~min}$ to desorb $\mathrm{Si}$ atoms and form a graphene layer.

\section{2. $\mathrm{MoSe}_{2}$ thin films}

The $\mathrm{MoSe}_{2}$ films are grown by codepositing high-purity Se (purity 99.999\%) and Mo (purity 99.95\%) atoms from a Knudsen cell and an electron-beam evaporator, respectively, while the graphene-covered $\mathrm{SiC}$ substrate is kept at $530 \mathrm{~K}$ for a duration of $10 \mathrm{~min}$. After that, the sample is annealed at $870 \mathrm{~K}$ for $10 \mathrm{~min}$ to crystalize the $\mathrm{MoSe}_{2}$ films. The flux ratio of $\mathrm{Se}$ and $\mathrm{Mo}$ is about 10:1, and the excess $\mathrm{Se}$ atoms are desorbed from the substrate during the growth.

\section{3. $\mathrm{Mo}_{6} \mathrm{Se}_{6}$ nanowires}

To form $\mathrm{Mo}_{6} \mathrm{Se}_{6}$ nanowires, the sample is further heated to $870 \mathrm{~K}$ in situ. During this process, the Se atoms at $\mathrm{MoSe}_{2}$ edges preferentially evaporate upon the annealing treatment, changing the stoichiometry of Mo and Se at the edges and driving a transition to the $\mathrm{Mo}_{6} \mathrm{Se}_{6}$ phase. Since the reaction occurs at the defined location, the straight edges of 
$\mathrm{MoSe}_{2}$ layers provide excellent templates for the growth of $\mathrm{Mo}_{6} \mathrm{Se}_{6}$ nanowires. By tuning the annealing time, we can control the ratio of $\mathrm{Mo}_{6} \mathrm{Se}_{6}$ terminated edges [Figs. S3(a)$\mathrm{S} 3$ (c) [28] ]. A minimum of $8 \mathrm{~h}$ is needed to fully saturate the $\mathrm{MoSe}_{2}$ edges with $\mathrm{Mo}_{6} \mathrm{Se}_{6}$ nanowires. Further extended annealing degrades partial $\mathrm{MoSe}_{2}$ layers and, meanwhile, forms some isolated $\mathrm{Mo}_{6} \mathrm{Se}_{6}$ nanowires connecting to $\mathrm{MoSe}_{2}$ islands at two ends [Fig. S3(d) [28] ]. These wires are tantalizing for making interconnects to the monolayer circuits.

\section{B. STM measurement}

The experiments are performed with a cryogenic custommade Unisoku STM [43]. An electrochemically etched W wire is used as the STM tip. Prior to measurements, the tip is characterized on a $\operatorname{Ag}(111)$ multilayer film grown on a $\operatorname{Si}(111)$ substrate, which is cleaned by several flashing cycles to $1500 \mathrm{~K}$. The tunneling spectra are obtained by lock-in detection of the tunneling current with a modulation voltage at $983 \mathrm{~Hz}$ feeding into the sample bias.

\section{DFT calculation}

The electronic structure and the DOS of $\mathrm{Mo}_{6} \mathrm{Se}_{6}$ nanowires are calculated in the framework of DFT within the generalized gradient approximation [44] and local density approximation, where both approximations deliver identical results. The projector-augmented-wave method implemented in the Vienna Ab Initio Simulation Package is employed with an energy cutoff of $500 \mathrm{eV}[45,46]$. A supercell with vacuum spaces of $20 \AA$ along the $x$ and $y$ directions is employed with a $1 \times 1 \times 9 k$-point mesh. The Phonopy [47] package is further used to calculate the phonon band structure using a $1 \times 1 \times 8$ supercell and $1 \times 1 \times 2 k$-point mesh. The calculations are performed at the Shanghai Supercomputing Center, the HPC Platform of ShanghaiTech University Library and Information Services and School of Physical Science and Technology.

All data needed to evaluate the conclusions in the paper are present in the paper and/or the Supplemental Material [28]. Additional data related to this paper may be requested from the authors. Correspondence and requests for materials should be addressed to Y. S. F. and J. T. L.

\section{ACKNOWLEDGMENTS}

The authors thank T. Hanaguri, H. W. Liu, X. Liu, and D. L. Feng for discussions. This work is funded by the National Key Research and Development Program of China (Grants No. 2017YFA0403501 and No. 2016YFA0401003), the National Science Foundation of China (Grants No. 11874161, No. 11522431, No. 11474112, No. 11774105, and No. 21873033), and Program for Professor of Special Appointment (Shanghai Eastern Scholar), JST CREST Grants No. JPMJCR1874 and No. JPMJCR16F1, and JSPS KAKENHI Grants No. 18 H03676 and No. 26103006, Japan.

The authors declare that they have no competing interests.

X. Y., J. J.X., L. Q., Z. M.Z., and W. H. Z. carried out the experiments. G. L. and J. T. L. did the DFT calculations. N.N. and J.T.L. constructed the theoretical modeling. Y. S. F., X. Y., J. J. X., J. T. L., and N. N. analyzed the data. Y.S. F. and N. N. wrote the manuscript with contributions from J. T. L. and G. L. All authors commented on the manuscript.

[1] T. Giamarchi, Quantum Physics in One Dimension (Oxford University Press, New York, 2003).

[2] G. Grüner, Density Waves in Solids (Addison-Wesley, Reading, MA, 1994).

[3] N. D. Mermin and H. Wagner, Absence of Ferromagnetism or Antiferromagnetism in One- or Two-Dimensional Isotropic Heisenberg Models, Phys. Rev. Lett. 17, 1133 (1966).

[4] P. Monceau, Electronic Crystals: An Experimental Overview, Adv. Phys. 61, 325 (2012).

[5] T. Aruga, Charge-Density Waves on Metal Surfaces, J. Phys. Condens. Matter 14, 8393 (2002).

[6] S. Smaalen, The Peierls Transition in Low-Dimensional Electronic Crystals, Acta Crystallogr. Sect. A 61, 51 (2005).

[7] G. Lee, H. Shim, J. M. Hyun, and H. Kim, Intertwined Solitons and Impurities in a Quasi-One-Dimensional Charge-Density-Wave System: In/Si(111), Phys. Rev. Lett. 122, 016102 (2019).

[8] S. Cheon, T. H. Kim, S. H. Lee, and H. W. Yeom, Chiral Solitons in a Coupled Double Peierls Chain, Science 350, 182 (2015).

[9] T. H. Kim, S. Cheon, and H. W. Yeom, Switching Chiral Solitons for Algebraic Operation of Topological Quaternary Digits, Nat. Phys. 13, 444 (2017).

[10] S. Brazovskii, C. Brun, Z.Z. Wang, and P. Monceau, Scanning-Tunneling Microscope Imaging of SingleElectron Solitons in a Material with Incommensurate Charge-Density Waves, Phys. Rev. Lett. 108, 096801 (2012).

[11] S. Brazovskii, Solitons: From Charge Density Waves to FFLO in Superconductors, Physica (Amsterdam) B404, 482 (2009).

[12] C. G. Zeng, P. R. C. Kent, T. H. Kim, A. P. Li, and H. H. Weitering, Charge-Order Fluctuations in One-Dimensional Silicides, Nat. Mater. 7, 539 (2008).

[13] S. Barja et al. Charge Density Wave Order in $1 D$ Mirror Twin Boundaries of Single-Layer $\mathrm{MoSe}_{2}$ Nat. Phys. 12, 751 (2016).

[14] F. J. Ribeiro, D. J. Roundy, and M. L. Cohen, Electronic Properties and Ideal Tensile Strength of MoSe Nanowires, Phys. Rev. B 65, 153401 (2002).

[15] D. Çakır, E. Durgun, O. Gülseren, and S. Ciraci, First Principles Study of Electronic and Mechanical Properties of Molybdenum Selenide Type Nanowires, Phys. Rev. B 74, 235433 (2006). 
[16] L. Venkataraman and C. M. Lieber, Molybdenum Selenide Molecular Wires as One-Dimensional Conductors, Phys. Rev. Lett. 83, 5334 (1999).

[17] L. Venkataraman, Y.S. Hong, and P. Kim, Electron Transport in a Multichannel One-Dimensional Conductor: Molybdenum Selenide Nanowires, Phys. Rev. Lett. 96, 076601 (2006).

[18] X. D. Xu, W. Yao, D. Xiao, and T.F. Heinz, Spin and Pseudospins in Layered Transition Metal Dichalcogenides, Nat. Phys. 10, 343 (2014).

[19] J. Kibsgaard, A. Tuxen, M. Levisen, E. Lægsgaard, S. Gemming, G. Seifert, J. V. Lauritsen, and F. Besenbacher Atomic-Scale Structure of $\mathrm{Mo}_{6} \mathrm{~S}_{6}$ Nanowires, Nano Lett. 8, 3928 (2008).

[20] D. Le, W. Lu, M. Aminpour, C. Wang, Q. Ma, T. S. Rahman, and L. Bartels Growth of Aligned $\mathrm{Mo}_{6} \mathrm{~S}_{6}$ Nanowires on $\mathrm{Cu}(111)$, Surf. Sci. 611, 1 (2013).

[21] Y. Yu, G. Wang, Y. Tan, N. Wu, X.-A. Zhang, and S. Qin Phase-Controlled Growth of One-Dimensional $\mathrm{Mo}_{6} \mathrm{Te}_{6}$ Nanowires and Two-Dimensional $\mathrm{MoTe}_{2}$ Ultrathin Films Heterostructures, Nano Lett. 18, 675 (2018).

[22] H. Zhu, Q. Wang, C. Zhang, R. Addou, K. Cho, R. M. Wallace, and M.J. Kim New $\mathrm{Mo}_{6} \mathrm{Te}_{6}$ Sub-NanometerDiameter Nanowire Phase from $2 \mathrm{H}-\mathrm{MoTe}_{2}$, Adv. Mater. 29, 1606264 (2017).

[23] X. Sang, X. Li, W. Zhao, J. Dong, C. M. Rouleau, D. B. Geohegan, F. Ding, K. Xiao, and R. R. Unocic In Situ Edge Engineering in Two-Dimensional Transition Metal Dichalcogenides, Nat. Commun. 9, 2051 (2018).

[24] W. Huang, X. Wang, X. Ji, Z. Zhang, and C. Jin, In-Situ Fabrication of $\mathrm{Mo}_{6} \mathrm{~S}_{6}$-Nanowire-Terminated Edges in Monolayer Molybdenum Disulfide, Nano Res. 11, 5849 (2018).

[25] J.H. Lin et al. Flexible Metallic Nanowires with SelfAdaptive Contacts to Semiconducting Transition-Metal Dichalcogenide Monolayers, Nat. Nanotechnol. 9, 436 (2014).

[26] Y. Zhang et al. Direct Observation of the Transition from Indirect to Direct Bandgap in Atomically Thin Epitaxial $\mathrm{MoSe}_{2}$, Nat. Nanotechnol. 9, 111 (2014).

[27] Q. Wang, W. Zhang, L. Wang, K. He, X. Ma, and Q. Xue Large-Scale Uniform Bilayer Graphene Prepared by Vacuum Graphitization of 6H-SiC(0001) Substrates, J. Phys. Condens. Matter 25, 095002 (2013).

[28] See Supplemental Material at http://link.aps.org/supplemental/ 10.1103/PhysRevX.10.031061 for Supplemental figures, details on the atomic resolution of the nanowires, temperature dependence of the CDW gap, phason-polaron theory, and influence on the CDW from the substrate, pinning and finite wire length, which includes Ref. [29].

[29] P. A. Lee, T. M. Rice, and P. W. Anderson, Conductivity from Charge or Spin Density Waves, Solid State Commun. 14, 703 (1974).

[30] M. M. Ugeda et al. Giant Bandgap Renormalization and Excitonic Effects in a Monolayer Transition Metal Dichalcogenide Semiconductor, Nat. Mater. 13, 1091 (2014).
[31] P. W. Anderson, Absence of Diffusion in Certain Random Lattices, Phys. Rev. 109, 1492 (1958).

[32] S. Qin et al. Correlating Electronic Transport to Atomic Structures in Self-Assembled Quantum Wires, Nano. Lett. 12, 938 (2012).

[33] D. F. Urban, C. A. Stafford, and H. Grabert, Scaling Theory of the Peierls Charge Density Wave in Metal Nanowires, Phys. Rev. B 75, 205428 (2007).

[34] E. J. Mueller, Review of Pseudogaps in Strongly Interacting Fermi Gases, Rep. Prog. Phys. 80, 104401 (2017).

[35] S. Brazovskii and S. Matveenko, Pseudogaps due to Sound Modes: From Incommensurate Charge Density Waves to Semiconducting Wires, Sov. Phys. JETP 96, 555 (2003).

[36] H. Ding, T. Yokoya, J. C. Campuzano, T. Takahashi, M. Randeria, M. R. Norman, T. Mochiku, K. Kadowaki, and J. Giapintzakis Spectroscopy Evidence for a Pseudogap in the Normal State of Underdoped High- $T_{c}$ Superconductors, Nature (London) 382, 51 (1996).

[37] B. Sacépé, T. Dubouchet, C. Chapelier, M. Sanquer, M. Ovadia, D. Shahar, M. Feigel'man, and L. Ioffe Localization of Preformed Cooper Pairs in Disordered Superconductors, Nat. Phys. 7, 239 (2011).

[38] U. Chatterjee et al. Emergence of Coherence in the ChargeDensity Wave State of $2 \mathrm{H}-\mathrm{NbSe}_{2}$, Nat. Commun. 6, 6313 (2015).

[39] P. A. Lee, T. M. Rice, and P. W. Anderson, Fluctuating Effects at a Peierls Transition, Phys. Rev. Lett. 31, 462 (1973).

[40] F. H. Long, S. P. Love, and B. I. Swanson, Evidence for Modification of the Electronic Density of States by ZeroPoint Lattice Motion in One Dimension: Luminescence and Resonance Raman Studies of an MX Solid, Phys. Rev. Lett. 71, 762 (1993).

[41] L. Perfetti, H. Berger, A. Reginelli, L. Degiorgi, H. Hochst, J. Voit, G. Margaritondo, and M. Grioni, Spectroscopic Indications of Polaronic Carriers in the Quasi-OneDimensional Conductor $\left(\mathrm{TaSe}_{4}\right)_{2} I$, Phys. Rev. Lett. 87, 216404 (2001).

[42] W. Jolie et al. Tomonaga-Luttinger Liquid in a Box: Electrons Confined within MoS2 Mirror-Twin Boundaries, Phys. Rev. X 9, 011055 (2019).

[43] L. Peng, Y. Yuan, G. Li, X. Yang, J.-J. Xian, C.-J. Yi, Y.-G. Shi, and Y.-S. Fu Observation of Topological States Residing at Step Edges of $\mathrm{WTe}_{6}$, Nat. Commun. 8, 659 (2017).

[44] J. P. Perdew, K. Burke, and M. Ernzerhof, Generalized Gradient Approximation Made Simple, Phys. Rev. Lett. 77, 3865 (1996).

[45] G. Kresse and J. Hafner, Ab Initio Molecular Dynamics for Liquid Metals, Phys. Rev. B 47, 558 (1993).

[46] G. Kresse and D. Joubert, From Ultrasoft Pseudopotentials to the Projector Augmented-Wave Method, Phys. Rev. B 59, 1758 (1999).

[47] A. Togo and I. Tanaka, First Principles Phonon Calculations in Materials Science, Scr. Mater. 108, 1 (2015). 